Cahiers de recherches médiévales

\title{
La bibliothèque de l'abbaye de Saint-Victor de Paris
}

Jean-Pierre Willesme

\section{(2) OpenEdition}

Journals

Édition électronique

URL : https://journals.openedition.org/crm/11525

DOI : $10.4000 / \mathrm{crm} .11525$

ISSN : $1955-2424$

Éditeur

Honoré Champion

Édition imprimée

Date de publication : 15 juin 2009

Pagination : 241-255

ISSN : 1272-9752

\section{Référence électronique}

Jean-Pierre Willesme, "La bibliothèque de l'abbaye de Saint-Victor de Paris », Cahiers de recherches médiévales [En ligne], 17 | 2009, mis en ligne le 15 juin 2012, consulté le 15 décembre 2022. URL : http://journals.openedition.org/crm/11525; DOI : https://doi.org/10.4000/crm.11525 


\title{
RM
}

\section{La bibliothèque de l'abbaye de Saint-Victor de Paris}

\begin{abstract}
As early as 1113, shortly after its founding, the Abbey of Saint-Victor in Paris included a library that grew steadily thanks to the copying of manuscripts. The old library, dedicated to manuscripts, was located in the building originally occupied by Bishop Arnoul of Lisieux, a friend of Saint-Victor. A new library was erected in the early $16^{\text {th }}$ century by the abbot Nicaise de l'Orme, and the manuscripts were chained to desks. In 1639, the gallery was decorated with portraits of the abbey's most renowned monks, beginning with its founder William of Champeaux. Jean Picard and Jean de Thoulouse were the abbey's most erudite canons in the seventeenth century. In 1651, the flooding of the Seine and the Biève leads to the library's being raised by a floor, and in 1652, an important donation of printed books by Henri du Boucher, counselor to the Paris Parlement, led to its expansion. In the mid-1 $18^{\text {th }}$ century, the abbey commissioned the architect Danjan to build a new and much larger library, which was was not completed until the eve of the Revolution, when the abbey was completely destroyed. Most books were transferred into the collections of the Bibliothèque nationale de France. The abbey may have been situated on the site of the Faculty of Science of la Halle aux vins (Wine Market), place Jussieu, which has since been renamed Faculté des sciences Paris-Centre.
\end{abstract}

Résumé : L'abbaye fut dotée presque dès son origine, au début du XII siècle, en 1113, d'une bibliothèque qui s'enrichissait par la copie des manuscrits. La vieille librairie, consacrée aux manuscrits, se trouvait dans la maison qu'avait habitée un ami de Saint-Victor, l'évêque Arnoul de Lisieux. Au début du XVI eiècle, l'abbé Nicaise de l'Orme élève une nouvelle bibliothèque. Les manuscrits consultés sont enchaînés sur des pupitres. En 1639, la galerie est dotée de tableaux représentant les hommes les plus illustres de l'abbaye depuis la fondation par Guillaume de Champeaux. Jean Picard et Jean de Thoulouse sont les chanoines les plus érudits du XVII siècle. Les graves inondations de la Seine et de la Bièvre, en 1651 obligent à monter d'un étage la bibliothèque, qui doit dans l'année suivante, en 1652, s'agrandir à la suite de l'important legs d'ouvrages imprimés par Henri du Boucher, conseiller au parlement de Paris. Au milieu du XVIII siècle, se trouvant trop à l'étroit, les religieux font appel à l'architecte Danjan pour se doter d'une nouvelle bibliothèque, qui ne peut ouvrir qu'à la veille de la Révolution.

Tous les bâtiments sont rapidement détruits avant de faire place à la Halle aux vins puis à la faculté des sciences de Jussieu.

L'étude ayant toujours été à l'honneur chez les victorins, l'abbaye possède une bibliothèque peu après sa fondation en $1113^{1}$. Des dons d'ouvrages ont lieu en 1133, puis 1140. En 1182, l'évêque Arnoul de Lisieux fait don de plusieurs

1 F. Gasparri, «Scriptorium et bureau d'écriture de l'abbaye Saint-Victor de Paris », Bibliotheca victorina, t. I, L'abbaye parisienne de Saint-Victor au Moyen Âge, communications réunies par J. Longère, Paris-Turnhout, Brepols, 1991, p. 119-139.

Cahiers de Recherches Médiévales, 17, 2009 
volumes ${ }^{2}$. Les dons se poursuivent au cours du XIII ${ }^{\mathrm{e}}$ siècle mais se raréfient au $\mathrm{XIV}^{\mathrm{e}}$ siècle. Les premiers ouvrages imprimés apparaissent à partir de la seconde moitié du $\mathrm{XV}^{\mathrm{e}}$ siècle.

Il s'agit en fait d'étudier les bibliothèques successives de Saint-Victor. Elles ont changé plusieurs fois d'emplacement. La chronologie et l'évolution topographique ne se laissent pas facilement appréhender.

\section{De la maison d'Arnoul de Lisieux à la bibliothèque de Nicaise Delorme}

La vieille librairie ${ }^{3}$, suivant la première dénomination, se trouve dans la maison qu'a habitée Arnoul de Lisieux, ami de l'abbaye au XII ${ }^{\mathrm{e}}$ siècle, mort en $1184^{4}$. Elle était consacrée à la bibliothèque des manuscrits, qui furent longtemps la grande et l'unique richesse de cette bibliothèque. Située au bord de la Bièvre, restaurée en $1531^{5}$, elle a sans doute conservé cet usage en partie jusqu'en $1663^{6}$, date où elle est détruite. Selon Franklin, la maison d'Arnoul de Lisieux se complétait d'un local attenant au cloître et situé entre l'église et le dortoir ${ }^{7}$.

Au début du $\mathrm{XVI}^{\mathrm{e}}$ siècle, entre 1501 et 1509 , à l'initiative de l'abbé Nicaise Delorme, la construction d'une nouvelle bibliothèque est lancée sous le contrôle du chambrier Guillaume Tupin ${ }^{8}$. Elle trouve place au-dessus de la nouvelle sacristie

\footnotetext{
${ }^{2}$ D'après A. Franklin, Les anciennes bibliothèques de Paris, Histoire générale de Paris, t. I, Paris, 1867, p. 138-142. Cet ouvrage, malgré quelques erreurs, reste la meilleure référence imprimée.

${ }^{3}$ Jean de Thoulouse, Annales regalis abbatice sancti Victoris parisiensis, BnF, ms. lat. 14373, fol. $481^{\mathrm{r}}$, année 1509. La vieille librairie (vetus libraria) s'identifie, tout au moins en partie, à la maison d'Arnoul, évêque de Lisieux, qui résida au bord de la Bièvre: Vetus libraria corruens evulsa fuit, et pulchrior et ornatior extructa fuit bibliotheca, in qua posmodum dispositi sunt codices [...]. Dans les Antiquitates regalis abbatice sancti Victoris parisiensis, BnF, ms. lat. 14677, fol. $35^{\mathrm{v}}$, Jean de Thoulouse, qui réutilise les anciennes chroniques, affirme qu'Arnoul de Lisieux avait élevé à Saint-Victor de très belles constructions de sa propre main : in domibus pulcherrimis quas ibi ad opus suum construxerat.

${ }^{4}$ Arnoul de Lisieux avait également fait bâtir une chapelle, qui prit le nom de chapelle de Pastourel (ou Pastoureaux) à la suite de la fondation d'une messe. Jean Pastourel fut premier président de la Cour des comptes de 1388 à 1392.

${ }^{5}$ Jean de Toulouse, Annales, op. cit., BnF, ms. lat. 14374, fol. $197^{\mathrm{r}}$. Thoulouse cite ici une inscription rédigée en vers par le poète victorin Jean Corrard, mort en 1546.

${ }^{6}$ Philippe Gourreau, BnF, ms. fr. 24082, fol. $284^{\mathrm{v}}$ et $286^{\mathrm{r}}$. Les « Mémoires » de Philippe Gourreau de la Proustière ont été publiés d'après BnF, ms. fr.24082 et 24083, par Béatrix de Buffévent, Paris et Île-de-France, Études et documents, Paris, 1990, p. 324.

${ }^{7}$ Suivant en cela Jean de Thoulouse ; Antiquitatum regalis abbatia sancti Victoris parisiensis, $\mathrm{BnF}$, ms. lat. 14677 , fol. $377^{\mathrm{V}}$ : Locus autem ille inter ecclesiam et dormitorium claustro contiguum vicinas habebat ades Arnlphi quondam lexoviensis episcopi apud nos demorantis anno 1181, quas post ejus obitum veterani abbates officio cedentes intrabitarunt nempe Ascelinus, Robertus, Joannes de Brueriis, et Gaufridus Pellegay; sed cum post tria sacula adificia vetustate corruerent, Nichasius de Lorme 33. abbas noster anno 1508 veterem bibliothecam in novum transtulit adificium sua cura extructum [...].

${ }^{8}$ Jean de Thoulouse, BnF, ms. lat. 14677, fol. 112r: Guillermus Tupin [...] novum aedificium manuscripta bibliotheca prout nunc extat adificari curavit.
} 
dépendant de l'église', et cette disposition est connue jusqu'en 1651. La nova libraria a désormais supplanté la vetus libraria. Les manuscrits sont enchaînés sur une cinquantaine de pupitres sur lesquels ils peuvent être consultés.

Cette bibliothèque est organisée par Claude de Grandrue qui, comme ce fut souvent le cas, joignait à ses fonctions de chantre celles de bibliothécaire. ${ }^{10}$ Il classe les manuscrits et les premiers livres imprimés ; il en rédige un catalogue, le dernier d'esprit encore médiéval ${ }^{11}$. Après lui, le plus célèbre est l'érudit Jean Picard, qui dirige l'école de Saint-Victor au début du XVII ${ }^{\mathrm{e}}$ siècle et publie divers ouvrages, notamment des commentaires sur saint Bernard ${ }^{12}$; épuisé par l'étude, il meurt prématurément dès janvier 1615. En 1639, le prieur Jean de Thoulouse, qui se flattait d'avoir été son élève, orne la bibliothèque de soixante-sept tableaux des hommes et religieux les plus illustres de la maison depuis son fondateur, Guillaume de Champeaux $^{13}$. Les évêques Arnoul de Lisieux et Maurice de Sully sont représentés d'après des sceaux conservés dans les archives de l'abbaye. D'autres portraits, dont celui du roi Louis le Gros, qui se déclarait aussi fondateur de la maison, sont peints d'après des descriptions de manuscrits. On y place aussi une copie d'un portrait du roi régnant, Louis XIII, au-dessus de la porte principale de l'entrée. On connaît même le nom du peintre, un certain Toutain, grâce aux notes de Philippe Gourreau de la Proustière, qui fut lui-même bibliothécaire et s'attribue l'initiative du décor, ayant aussi la «charge de cellérier, dont le sujet est de faire la despence de la maison $\gg^{14}$. Le local est décrit tardivement, en 1649, par Dubuisson-

${ }^{9}$ Ibid., fol. $380^{\mathrm{r}}$ : Et anno 1508 scribet [Pierre Boucher] : eodem anno (inquit) adificata est nova libraria in domo nostra sancti Victoris, et sacristia sub ea, et quasi totus ille locus renovatus est totaliter expensis ecclesia. Jean de Thoulouse, qui cite toujours ses sources, utilise ici le journal rédigé par le chanoine Pierre Boucher au début du XVI e siècle, journal qu'il juge d'un style relâché.

${ }^{10}$ Jean de Thoulouse place Guillaume Tupin et Claude de Grandrue dans le chapitre des restaurateurs de la bibliothèque, chapitre LXXI, ibid., fol. $112^{\mathrm{r}}$ et $112^{\mathrm{v}}$.

${ }^{11}$ Ce catalogue a été publié : Le catalogue de la bibliothèque de l'abbaye de Saint-Victor de Paris de Claude de Grandrue, 1514, édition du Centre national de la recherche scientifique, Paris, 1983. La publication est précédée d'une utile introduction par G. Ouy : «Historique de la bibliothèque de Saint-Victor », I-LXI. On y apprend qu'un certain nombre de livres ne devaient jamais quitter l'infirmerie, où ils instruisaient les malades dans l'art de bien mourir. Sur la catalogue que nécessitait la nouvelle bibliothèque, ibid., p. 2.

${ }^{12}$ Jean de Thoulouse, ibid., ms. lat. 14677, fol. $118^{\mathrm{r}}$.

${ }^{13}$ Le Mémorial de Jean de Thoulouse, prieur-vicaire de Saint-Victor de Paris, édition et présentation par J.-B. Capit, t. II, Les années 1638-1659, Turnhout, Brepols, (Bibliotheca victorina XIII), 2008, p. 75-76.

${ }^{14}$ Sur le décor de la bibliothèque et sur le peintre Pierre Toutain (1645-1686), voir Gourreau de la Proustière, op. cit., 1990, p. 57 : «Je persuadé au P. Thoulouse de faire paindre les hommes illustres de la maison en dignité, science et piétté, ce qu'il ambrassast aisément, estant fort porté pour l'antiquité dont il scavoit l'histoire. Un pintre nommé Toutain nous fit tous les portrais que l'on void encore au-dessus des tablettes de la bibliotèque, le P. Thoulouse fournissant les noms, les dignitées et la façon des habits [...]. Il y avoit quantités de petittes fenestres entre chacune desquelles il y avoit un de ces tableaux avec des ornemens autour en camaieux gris. » 
Aubenay ${ }^{15}$. La forme de la bibliothèque est comparée à celle d'une potence. La bibliothèque n'est pas encore publique, bien qu'elle ait pu être ouverte à certains savants ou à quelques personnes amies des religieux. Les livres n'étaient pas tous conservés dans la grande bibliothèque. Quant aux manuscrits liturgiques, ils étaient conservés à l'église dans la bibliothèque du chœur, sous la responsabilité du chantre. Au milieu de XVII ${ }^{\mathrm{e}}$ siècle commencent les grands remaniements et les constructions nouvelles. Jean de Thoulouse, dans son Mémorial signale une inondation en janvier 1649, si bien qu' «il fallut la nuit du mercredy octave des Roys après matines desloger promptement nos jeunes frères qui estoient au noviciat soubz la grande bibliothèque $»^{16}$. En janvier 1651, c'est une inondation de grande ampleur qui perturbe la vie des victorins ${ }^{17}$; la bibliothèque est cette fois envahie par les eaux de la Seine, qui débordent brusquement. Il faut surélever le bâtiment qui l'abrite ; les collections sont alors installées au deuxième étage et rangées dans près de mille deux cents armoires. Le noviciat succède à la bibliothèque dans les locaux du premier étage devenus disponibles. Les tableaux, déjà dégradés, sont déplacés. Il ne reste, un temps, dans le noviciat, qu' un « tableau d'un religieux qui fait mine de monstrer à un ecclésiastique la bibliothèque ${ }^{18}$

\section{Le legs Du Bouchet et le réaménagement de la bibliothèque}

Un fait important se produit : par testament du 27 mars 1652, Henri Du Bouchet, seigneur de Bournonville, conseiller au Parlement de Paris, lègue toute sa bibliothèque à l'abbaye de Saint-Victor, qui s'enrichit ainsi de livres imprimés. On doit de ce fait réaliser un escalier de pierre de taille au-dessus du noviciat, que l'architecte Robert Boudin ${ }^{19}$ construit pour monter à la nouvelle salle réaménagée. Du Bouchet exige dans son testament que le public ait libre accès aux collections trois jours par semaine et que, chaque année, des avocats généraux du Parlement contrôlent l'état de la bibliothèque et des ouvrages légués en particulier. Le bibliothécaire Eustache de Blémur contribue beaucoup à l'application des volontés exprimées par Du Bouchet. On élève ensuite un grand pavillon au bout de la bibliothèque pour y mettre les manuscrits, désormais mis à l'écart des livres imprimés et quelque peu délaissés. À cette occasion, de nombreuses démolitions de vieux bâtiments ont lieu, dont les matériaux sont utilisés pour cette nouvelle

15 F.-N. Baudot Dubuisson-Aubenay, Paris et son diocèse. Itinéraire par Monsieur Dubuisson-Aubenay, gentilhomme d'honneur chez Monsieur Du Plessis, Bibl. Mazarine, ms. 4404, fol. $145^{\mathrm{r}}$. En 1649, cet auteur signale que c'est «une autre galerie séparée » qui renferme la plupart des livres imprimés.

${ }^{16}$ Le Mémorial de Jean de Thoulouse, op. cit., t. II, p. 257-258.

${ }^{17}$ Ibid., p. 313-314.

${ }^{18}$ Gourreau de la Proustière, Mémoires, op. cit., 1990, p. 57.

${ }^{19}$ Jean de Thoulouse, BnF, ms. fr. 24081, p. 31, année 1653. Il fallut huit mois de travail pour réaliser cet escalier, terminé le 24 novembre 1653 . Boudin avait le titre de maitre maçon et devait être originaire de Rouen. Il avait également bâti une nouvelle rose dans l'église de Saint-Victor. On le trouve dans les travaux d'une sacristie au prieuré de Villiers-le-Bel. Voir Gourreau de la Proustière, op. cit., 1990, p 146 : «J'ay fait faire la sacristie qui donne sur la rue, l'ancienne estant trop pettite et incommode, en 1652 ; maistre Boudin, maçon de Paris, conduisoit l'ouvrage ». 
construction. On démolit, selon les Mémoires de Gourreau, la chapelle des Pastoureaux et la maison d'Arnoul de Lisieux, ainsi qu'une petite bibliothèque des livres imprimés « dans laquelle on descendoit du bout de dortoir tirant vers le pré ». On en profite pour couvrir la Bièvre et y établir une terrasse ${ }^{20}$. Tous les vieux bâtiments disparus, proches des dortoirs, sont remplacés par un jardin ${ }^{21}$.

En 1684, la bibliothèque renfermait déjà 18000 volumes et 3000 manuscrits ${ }^{22}$. À cette même date, un dessin de Nicolas Guérard, qui suit une visite de contrôle des avocats généraux, montre l'intérieur de la bibliothèque dans son nouvel aménagement du deuxième étage ${ }^{23}$. La salle de lecture, couverte d'un plafond lambrissé ${ }^{24}$, est éclairée par des fenêtres à vitres losangées. Les livres sont contenus dans des placards. Sous le plafond, court une frise de portraits dans des encadrements rectangulaires séparés par des pilastres. Ces tableaux, connus depuis 1639, puis déplacées, venaient sans doute d'être restaurés. Les lecteurs sont assis sur des tabourets, devant de petits pupitres dans l'embrasure des fenêtres. La salle de lecture offre cinquante places assises. Ce document était destiné à prouver au roi que la bibliothèque était très fréquentée par les étudiants. À partir du milieu du XVIII ${ }^{\mathrm{e}}$ siècle, la bibliothèque est désormais ouverte au public de huit à dix heures le matin et de deux à quatre heures le soir, jusqu'à cinq heures même en été, les lundis,

\footnotetext{
${ }^{20}$ Gourreau de la Proustière, op. cit., 1990, p. 324 : «Et par cette bibliotèque - la chappelle des pastoureaux fesoit un coude dedans cette petitte bibliotèque - on passoit dans le logement de l'evesque Aroud, plain de manuscrips, qui nous menoit en tournant dans le reste de la bibliotèque des manuscrips qui terminoit vers l'église qui fait maintenant le noviciat ayant eslevé le corps de logis pour mettre la bibliothèque des imprimez au-dessus. Tout cela, avec fort peu d'aultres pierres, nous a servy à bastir un grand pavillon au bout de la bibliotèque pour mettre nos manuscripts ». Ces faits se situent sans doute autour de 1660, bien que le contexte se rattache aux trois années de Gourreau à Saint-Victor (1663-1666).

${ }^{21}$ Gourreau de la Proustière, op. cit., 1990, p 326-327 : «Ce n'est plus qu'un jardin de toutte cette estendue là, bornée par les grilles de fer de la terrasse, en sorte qu'on ne diroit pas qu'il y eust eu en cet endroit tant de bastimens comme de celuy d'Arnould, d'une chapelle et d'une bibliothèque ».

${ }^{22}$ G.-L. Lerouge, Curiosités de Paris, de Versailles, Marly, Vincennes, S. Cloud et des environs, nouvelle édition augmentée, Paris, 1771, t. I, p. 394. Cette bibliothèque est «une des plus nombreuses et des plus parfaites de Paris, en livres rares et manuscrits très anciens et curieux ». Voir A. Masson, Le décor des bibliothèques du Moyen Âge à la Révolution, Genève, 1972, p. 106-108, fig. 58, p. 195.

${ }^{23}$ Une séance de lecture à la bibliothèque de Saint-Victor en 1684, Arch. nat., fonds Le Peletier, 259 AP 109, pièce annexe $n^{\circ} 2$. Nicolas Guérard (né vers 1648-mort en 1719) était aussi un graveur installé rue Saint-Jacques.

${ }^{24}$ Jean de Thoulouse, BnF, ms. lat. 14677 , fol. $377^{\mathrm{V}}$. La bibliothèque, suivant le règlement, doit être intérieurement garnie de boiseries, de peur que l'humidité des parois ne se communique aux manuscrits et les détériore: Ipsa autem armaria intrinsecus ligno vestita esse debent, ne humor parietum membranas rubigine aliqua, sive humectatione aliqua inficiat. Les règlements de la bibliothèque concernant les livres eux-mêmes dépassent le cadre de cet article. Les aménagements de la bibliothèque furent toujours plus austères que ceux de sa voisine Sainte-Geneviève.
} 
mercredis et samedis ${ }^{25}$. Le lecteur y est bien accueilli. Quand les jours sont fêtés, l'ouverture est reportée au lendemain. La bibliothèque était chaque année fermée pendant deux mois, du 15 août au 18 octobre $^{26}$. Le bibliothécaire est toujours un religieux de la maison. Quant aux manuscrits liturgiques, ils étaient conservés à l'église dans la bibliothèque du chœur, sous la responsabilité du chantre.

\section{À la veille de la Révolution, "l'affaire de la bibliothèque »}

Dans la deuxième moitié du XVIII siècle, les victorins, dans l'idée d'un plan de rénovation plus vaste ${ }^{27}$, veulent se doter d'une nouvelle et belle bibliothèque, en retour d'angle par rapport au noviciat. Les acquisitions d'ouvrages ont été importantes si bien qu'un bâtiment nouveau est indispensable pour agrandir la bibliothèque et mieux accueillir les gens de lettres. Dès 1764, les tractations apparaissent au cours des réunions du chapitre. Le 23 mars, une visite de l'architecte Danjan est demandée $e^{28}$. Il s'ensuit de nombreuses et fréquentes délibérations autour de « l'affaire de la bibliothèque ${ }^{29}$. Dans ces négociations, Mgr Jarente de La Bruyère, évêque d'Orléans, proche de Choiseul, chargé de la feuille des bénéfices, sert d'intermédiaire et d'interlocuteur direct entre le roi et les victorins ${ }^{30}$. Le chapitre se réunit le 26 janvier 1774 en constatant l'impossibilité de suivre les premiers plans dressés pour l'aile nouvelle à cause de la modicité de la somme accordée par le roi. On doit alors se rabattre sur le dernier plan proposé, qui prévoit un bâtiment d'une longueur inférieure de vingt-cinq pieds environ. Ce plan est agréé par le directeur des économats, M. de Marville, chargé de l'inspection et de l'exécution de la bibliothèque $^{31}$. Fin avril 1774, le prieur propose, pendant les travaux, de protéger manuscrits et livres dans la chambre de l'infirmerie. Le bibliothécaire est chargé d'y veiller avec le meilleur ordre possible. La pendule ${ }^{32}$, récemment léguée et prévue

${ }^{25}$ G.-L. Lerouge, Curiosités de Paris [...], op. cit., éd. 1716, p. 181. Dans la première partie du XVIII ${ }^{\mathrm{e}}$ siècle, elle est ouverte de $7 \mathrm{~h}$ à $11 \mathrm{~h}$ le matin.

${ }^{26}$ D' après A. Franklin, op. cit., t. I, 1887, p. 165.

${ }^{27}$ Ce plan de rénovation reposait sur la transformation du chœur de l'église.

${ }^{28}$ C'est essentiellement grâce à un registre capitulaire couvrant la période 1762-1789 que la vie intérieure de l'abbaye est connue. Arch. nat., LL 1451. Voir pièce annexe $n^{\circ} 3,20$ juillet 1764.

${ }^{29}$ Arch. nat., LL 1451 , fol. $12^{\mathrm{v}}, 13^{\mathrm{v}}, 14^{\mathrm{r}}, 15^{\mathrm{r}}, 17^{\mathrm{r}}, 17^{\mathrm{v}}, 18^{\mathrm{r}}, 18^{\mathrm{v}}, 22^{\mathrm{r}}, 22^{\mathrm{v}}, 23^{\mathrm{r}}, 39^{\mathrm{v}}, 113^{\mathrm{r}}, 119^{\mathrm{v}}$, $138^{\mathrm{r}}, 222^{\mathrm{v}}, 238^{\mathrm{r}}, 289^{\mathrm{r}}$.

${ }^{30}$ Louis Sextius de Jarente de la Bruyère, proche de Choiseul. Il éclaire le roi pour le choix des évêques et des abbés, d'où son titre de ministre de la Feuille [des bénéfices ecclésiastiques]. Il fut évêque de Digne, puis Orléans (1706-1788).

${ }^{31}$ Les économats concernent l'administration d'une abbaye pendant sa vacance abbatiale. Ce fut le cas entre les abbés commendataires François de Fitz-James, évêque de Soissons, décédé le 19 juillet 1764, et Antoine de Malvin de Montazet, archevêque de Lyon, nommé le 17 octobre 1764.

${ }^{32}$ Cette pendule de style Louis XIV est bien connue. Elle est aujourd'hui conservée à la bibliothèque de l'Arsenal dans le salon de musique de la duchesse du Maine (le régulateur est signé Gilles Martinot). Elle avait été offerte aux victorins en 1765 par Marguerite-Catherine Boucher, veuve de François-Louis Martinot-Duplessis. Le prieur Antoine Lagrenée la gardait 
pour le nouveau bâtiment, sera déposée provisoirement dans l'appartement du prieur. Le 3 juin 1777, l'architecte Danjan ayant fait part de ses réserves sur le projet d'un plancher, les chanoines demandent une expertise et enverront « le résultat de cette consultation à $\mathrm{M}$. de Marville afin de l'engager à se conformer aux conventions portées dans l'adjudication ${ }^{33}$.

Le 14 avril 1785, le chapitre décide de confirmer au bibliothécaire une exemption de la plupart des offices, dans l'idée d'une plus grande disponibilité «pour ce qui concerne le soin des livres et la manière de les communiquer soit à nos confrères soit aux étrangers, objets sur lesquels il a paru indispensable de former au plutôt les dispositions les plus précises ». Ces mesures sont prises en vue d'un meilleur accueil du public lors de la réouverture ; il faut aussi mettre en place des règlements sûrs et invariables.

Le 5 mai 1788 , le prieur «a fait lecture à la compagnie d'un mémoire à présenter pour obtenir des fonds nécessaires à l'entière perfection de notre bibliothèque ». Le prieur, le chambrier et les deux bibliothécaires feront les démarches indispensables auprès des ministres. Le 18 février 1789, Mgr de Fontanges, archevêque de Toulouse, est nommé abbé de Saint-Victor à la suite du décès de son prédécesseur, Malvin de Montazet $^{34}$. Le Roi en profite pour gratifier la bibliothèque d'une somme de cent cinquante mille francs à prendre sur le revenu de l'abbaye. Le registre capitulaire marque l'intérêt constant porté à la bibliothèque jusque dans ses dernières pages. Le 9 juin 1789, le chapitre, à la réception d'une lettre de M. de Brou, directeur général des économats, doit choisir entre achever la nouvelle bibliothèque ou réparer l'ancienne ; le vœu des chanoines est d'achever d'abord le nouveau bâtiment. Ėlevée sous la direction de Pierre-Alexandre Danjan ${ }^{35}$, la bibliothèque se compose désormais d'un bâtiment unique de quarante-huit mètres, terminé à ses extrémités par un pavillon formant saillie. D'après le Guide de Thiéry $^{36}$, toute la façade était ornée de balcons, de chambranles et de corniches exécutés avec goût : "Monsieur Danjan, architecte, vient de construire un nouveau bâtiment pour placer cette bibliothèque ; il a 24 toises de longueur sur 45 pieds de haut et il est terminé à chaque bout par un pavillon en avant-corps : la partie du milieu en arrière-corps est éclairée par cinq croisées ornées de bandeaux et frises, et chaque pavillon par une croisée ornée de chambranles couronnés de corniches avec

encore dans son appartement à la Révolution. Voir A. Franklin, op. cit., t. I, 1887, p. 163, note 3.

${ }^{33}$ Registre capitulaire ; Arch. nat., LL 1451, $138^{\mathrm{r}}$.

${ }^{34}$ François de Fontanges, archevêque de Toulouse (1788-1802). Voir Abbé G. Cayre, Histoire des évêques et archevêques de Toulouse depuis la fondation du siège jusqu'à nos jours, Toulouse, 1873.

${ }^{35}$ Sur cette dynastie d'architectes, voir M. Gallet, Les architectes parisiens du XVIII ${ }^{e}$ siècle, Mengès, 1995, p. 168. Domiciliés rue du Fouarre, ils furent de père en fils architectes de l'abbaye de Saint-Victor. Pourtant, le 7 février 1772, le chapitre des chanoines, marquant son indépendance, avait porté son choix sur un certain J.-B. Martial Poulain comme architecte de la maison. Le 20 juin 1778, Danjan est de nouveau réintroduit par l'abbé Malvin de Montazet. Voir Arch. nat., LL 1451, fol. $102^{\mathrm{v}}$ et $144^{\mathrm{v}}$.

${ }^{36}$ Luc-Vincent Thiéry, Guide des amateurs et des étrangers voyageurs à Paris, t. II, Paris, 1787, p. 162-163. 
des balcons saillants. Cet édifice, coiffé à l'italienne, est couronné d'un entablement avec consoles. L'intérieur n'étant point fini, le public se trouve privé depuis quelques années de la jouissance de cette belle bibliothèque ; il désire avec ardeur voir terminer un bâtiment dont l'objet est aussi utile ». Le premier étage renfermait les livres ; au second, dans un grand bâtiment contigu à l'infirmerie, se trouvaient les cartes géographiques et les médailles. Le service public ne peut reprendre qu'en 1788, à la veille de la Révolution qui voit la disparition complète de l'abbaye. En 1786, Lespinasse, dans une vue de Paris prise d'un belvédère de la rue des Boulangers et conservée au musée Carnavalet (Paris), dessine minutieusement cette aile, caractérisée par son toit très surbaissé ${ }^{37}$.

Les scellés sont apposés en février 1791 sur la bibliothèque. La plupart des ouvrages passent par le dépôt de la Pitié et entrent dans leur presque totalité à la Bibliothèque nationale ${ }^{38}$. Sur l'emplacement de l'ancienne abbaye, les arcades du 4 , rue Linné et du 6, rue Cuvier, encore visibles dans des cours d'immeubles, sont les restes de bâtiments annexes ${ }^{39}$.

Reste la question du repérage de ces lieux sur les vues de l'abbaye. La présence de la Bièvre, que les victorins avaient pourtant détournée volontairement sur leurs terres, a rendu difficile l'extension des bâtiments abbatiaux. C'est à sa proximité que les remaniements ont été les plus sensibles. Au XVII ${ }^{\mathrm{e}}$ siècle, les eaux avaient fini par devenir un foyer d'infection.

Trois côtés du grand cloître dataient encore du Moyen Âge. On y ensevelissait les religieux. Les dortoirs se trouvaient au chevet de l'église et furent toujours proches de la bibliothèque ; la place de ceux-ci a certainement bougé mais ils ne paraissent pas loin de la chapelle d'Arnoul de Lisieux au début du XVI ${ }^{\mathrm{e}}$ siècle. La chambre dite de l'abbé était à l'extrémité du dortoir à côté de la porte menant à la bibliothèque ${ }^{40}$. Il est difficile de repérer la trace de la vieille maison d'Arnoul de Lisieux. A la fin de l'Ancien Régime, on ne voit plus que la vaste chapelle de l'infirmerie sur un terrain du sud-est où la Bièvre est canalisée.

La nouvelle sacristie était contiguë à l'église. Le grand dortoir était situé à l'étage dans un bâtiment dont une partie était attribuée aux écoles ; il s'étendait vers le nord-est dans le prolongement de la galerie méridionale du grand cloître.

À partir du XVII ${ }^{\mathrm{e}}$ siècle, le noviciat eut aussi son destin étroitement lié à celui de la bibliothèque. À la Révolution, le noviciat se trouve toujours au premier étage d'une aile donnant sur le cimetière de l'abbaye. Il est certain qu'aucune vue ou plan

\footnotetext{
${ }^{37}$ Musée Carnavalet, Vue intérieure de Paris prise du belvédère de la maison de M. Fournel, par Louis Nicolas de Lespinasse, dépôt du Louvre à Carnavalet, IED 5367.

${ }^{38}$ A Franklin, op. cit., t. I, 1867, p. 166-167.

${ }^{39}$ J.-P. Willesme, «L'abbaye Saint-Victor de Paris sous la Révolution et la dispersion de son patrimoine », Bulletin de la société de l'histoire de Paris et de l'île-de-France, 1979, p. 150151.

${ }^{40}$ Jean de Thoulouse, Annales, BnF, ms. lat. 14374, fol. 51v, année 1515 : Hoc ipso anno post festum sancti Guinaili, destructa est in domo S. Victoris camera, qua dicebatur camera abbatis sita in extremitate dormitori juxta ostium dirigens ad bibliothecam.
} 
ancien de l'abbaye ne permet de situer les bibliothèques dans le cours de leur évolution. Les données des victorins sont elles-mêmes peu rigoureuses.

Jean-Pierre Willesme Musée Carnavalet, Paris 


\section{PIECES ANNEXES}

\section{LA BIBLIOTHEQUE EN 1639.} $75-76$

Citer ou renvoyer à l'Édition de Jean.-Baptiste. Capit, op. cit., t. II, 2008, p.

\section{VISITE DE LA BIBLIOTHEQUE EN 1684}

Arch. nat. 259 AP 109, dos. 5, archives Rosanbo, fonds Le Peletier.

Visite de la bibliothèque de Saint-Victor le 31 juillet 1684 par les avocats généraux Denis Talon et François Chrétien de Lamoignon en vertu du testament de Monsieur du Bouchet ${ }^{41}$ (un dessin de Nicolas Guérard, qui suit ce texte, montre le nombre important de lecteurs assidus dans la bibliothèque).

L'an mil six cens quatre vingts quatre le lundy trente un jour de juillet nous Denis Talon et Crestien François de Lamoignon conseillers ordinaires du Roy en son conseil d'Estat et ses advocats généraux en son parlement nous sommes transportez dans l'abbaye royalle de Saint-Victor pour y faire la visite que nous sommes invitez d'y faire chaque année de la bibliotèque publique par le testament de Monsieur du Bouchet, conseiller au parlement, voir et examiner l'usage qui s'en fait, la manière dont le public et particullièrement les pauvres estudians $\mathrm{s}^{42}$, y sont servis, l'employ qui se fait des revenus qui y sont destinez et les besoins auxquels on pouvoit pourvoir, pour ce qui est de l'usage, nous avons trouvé en entrant dans ladite bibliotèque un fort grand nombre d'estudians qui travailloient chacun sur quelque livre dans un grand ordre et fort profond silence, ce que l'on nous a assuré s'observer tousjours de la mesme manière, et que plusieurs mesme y passoient d'ordinaire les journées entières, y venant le matin et l'après disnée. Ce qui nous a persuadé de plus en plus non seulement de l'utilité mais encore de la nécessité de lad. bibliotèque pour le public lorsque nous avons considéré le besoin qu'un si grand nombre de personnes avoit de livres pour les aller chercher dans un lieu aussi éloigné et pour y travailler avec autant d'assiduité.

Estant ensuite entrez dans le grand cabinet qui est au bout de la bibliotèque nous aurions fait faire la lecture du testament de feu Monsieur du Bouchet conseiller au parlement en ce qui concerne le don qu'il a fait de sa bibliotèque à lad. abbaye pour le service du public, et la prière qu'il nous a faite de veiller par une visite chaque année à ce que les choses qu'il a prescrites pour ce sujet fussent observées, ce qu'ayant examiné nous aurions trouvé que selon ses intentions d'abord et à

${ }^{41}$ Dans son testament daté du 27 mars 1652, qui léguait sa riche bibliothèque à l'abbaye, Du Bouchet, conseiller au Parlement de Paris, plaçait sa donation sous la surveillance du Parlement lui-même et demandait aux avocats généraux du Roi de se rendre une journée par an à la bibliothèque afin d'examiner avec les Victorins l'état de la bibliothèque, désormais ouverte au public. Suivant ses volontés, il fut enterré à Saint-Victor, dans une des chapelles de l'église. Voir A. Franklin, op. cit., Paris, 1867, p. 154-157 et 180-182.

${ }^{42}$ Cette formulation, qui est une marque de charité, est héritée du Moyen Âge et reprise encore dans le legs du Bouchet (on parle d'écolier ou d'étudiant pauvre). 
l'entrée de la bibliotèque son portrait y est exposé en marbre accompagné de part et d'autre de deux inscriptions contenant l'une son éloge et le don qu'il a fait à ladite abbaye de sa bibliotèque pour l'usage du public, l'autre les lois par lesquelles il a pourveu à sa conservation, et ensuite nous avons remarqué que tous ses livres sont marquez de ses armes, gardez dans lad. bibliotèque sous une seule clef, et renfermez dans des armoires à jour d'où l'on nous a certifié qu'on ne les tire que pour les administrer à ceux qui viennent travailler dans ladite bibliotèque sans jamais les prester n'y sortir en dehors ; à cet effet nous avons appris que ladite bibliotèque est ouverte trois jours par sepmaine sçavoir les lundy, mercredy et samedy trois heures le matin et quatre heures l'après disnée, et que l'un des chanoines de lad. abbaye député à la garde de lad. bibliotèque s'y trouve et est tousjours présent avec un domestique entretenu et gagé par lad. abbaye pour administrer à tous ceux qui tiennent les livres qu'ils demandent les retirer ensuite de leurs mains quand ils en ont fait [lecture], et les resserrer chacun à leur place dans lesdites armoires, mais monobstans tous ces soings le bibliotécaire nous auroit remontré et fait plainte que, parmy le grand nombre de personnes qui viennent dans lad. bibliotèque, il ne peut empecher que quelques fois on ne luy emporte quelques livres ou que l'on en dechire quelques uns pour en emporter les feuilles comme il nous a fait voir en quelques volumes ; à l'esgard enfin de l'employ des rentes délaissées par led. Sieur du Bouchet pour l'entretien de lad. bibliotèque nous auroit remontré qu'il avoit laissé sept cent livres de rente de différente nature sur l'hostel de ville de Paris, partie pour dédommager l'abbaye des absences d'un des chanoines d'icelle pour veiller à la garde de ladite bibliotèque et au service du public et partie pour achepter les livres nouveaux et faire relier ou renouveller ceux qui viendroient à s'user et que tant que lesd. rentes ont esté remises entièrement sans en rien retenir pour lad. abbaye, on en avoit employé une partie à achepter de livres nouveaux dont la bibliotèque avoit esté augmentée de beaucoup pendant les premières années, et l'autre partie avoit esté employée à l'entretien et gages d'un domestique pour seconder et soulager le bibliotécaire dans le service du public, ce qui estoit absolument necessaire pour la conservation des livres veu le grand nombre de cens et deux cens personnes qui entrent chaque jour dans lad. bibliotèque mais que depuis les retranchemens qui sont survenus aud. rentes et la conversion de ce qui en restoit elles sont réduites à presens à trois cent douze livres seulement ce qui suffit à peyne à l'entretien et gages dud. domestique, de sorte qu'il ne reste aucun fonds n'y pour achepter les livres nouveaux n'y pour reparer ou renouveller les anciens qui viennent à s'user à quoy il nous a esté adjouté par le grand prieur et les officiers de lad. abbaye que, comme la partition des biens d'icelle entre la manse abbatialle et la manse conventuelle on ne leur avoit laissé de bien que ce qui estoit necessaire pour leur entretien. ils ne pouvoient pas faire de grandes espargnes sur leur revenu pour leur bibliotèque. Touparticullièrement que pour la loger dans un lieu propre à la comodité publique ils s'étoient engagés en de grandes dépences de bastimens pour lesquels ils avoient esté obligés d'emprunter de l'argent dont ils debvoient et payoient encor mil livres de rente et que tout ce qu'ils avoient pu prendre de plus sur eux les années dernières avoit esté pour faire relier de nouveau une partie des anciens manuscrits de lad. bibliotèque, ce qu'ils estoient encor obligez de continuer pour empecher la ruyne inévitable de cette partie principalle de leur bibliotèque qui fait une des curiosités de l'Europe, ce que nous avons jugé fort raisonnable, mais 
nous aurions en mesme temps reconnu le besoin pressant que l'on avoit de quelques secours pour soutenir un establissement si utile dont le public, soit pour avoir les livres nouveaux, soit pour réparer ou renouveller les anciens qui s'usent sans quoy ladite bibliotèque ne seroit pas dans peu de temps de grande utilité pour le public, et après avoir considéré les moyens les plus prompts et plus faciles que l'on pouvoit prendre, nous aurions esté d'avis que lesdicts de Saint-Victor remontrassent au Roy et à Monsieur le chancellier par un placet ${ }^{43}$ les besoins cy dessus et les supliant de leur accorder le don d'un exemplaire de chaque livre nouveau qui s'imprimera dans la suite et tel fonds qu'il plaira à sa Majesté leur ordonner pour subvenir aux despences necessaires, et par les plaintes qui nous ont esté faites par lesdits chanoines contre ceux qui emportent ou dechirent les livres, nous les aurons excitez de redoubler leurs soins, et en cas qu'ils surprennent quelqu'un dans de pareils deffauts, de faire executer contre eux l'arrest qui a esté rendu au parlement sur la mesme plainte et nous avons reytéré les deffences par nous cy devant faites conformément aux intentions dud. Sieur du Bouchet de sortir aucun des livres qui viennent de luy du lieu de lad. bibliotèque soit pour les prester au dehors soit mesme pour les porter dans les chambres desdits de Saint-Victor, attendu qu'estant destinez au service du public ils doibvent estre tousjours dans leur place touts prets à estre fournis à ceux qui le viennent demander, comme aussi de prester plusieurs volumes à la fois à lad. bibliotèque à une mesme personne mais seulement l'un après l'autre pour éviter les surprises de ceux qui, en sortant et rendant un livre, pouvoient en emporter un autre, sauf ausdits de Saint-Victor, d'user comme il leur plaira des livres de leur maison qui sont dans la mesme bibliothèque et qu'ils veullent bien rendre publics comme les autres, dont et de tous ce que dessus Nous avons ordonné estre dressé notre présent procez verbal que nous avons signé le jour et an que dessus.

Signé Talon,

De Lamoignon

Collationné à l'original Ce fait rendu par les conseillers du Roy notaires gardenottes du Roy nostre Sire au Chatelet de Paris Soubsignez l'an mil six cens quatre vingts quatre le quinziesme jour de décembre.

Torinon

\section{[Le document qui suit reprend les mêmes éléments adressés au Roi.]}

Les bibliotèque et chanoines de l'abbaye de Saint-Victor

[ mention ] : néant

Demandent

qu'il plaise au Roy leur accorder le don d'un exemplaire de chaque livre nouveau qui s'imprimera à l'advenir, et qu'à cet effet il soit fait mention de ce don dans toutes les lettres de privilèges qui s'expédieront au sceau à l'advenir,

le droit de franchise sur le sel et le vin nécessaire pour l'entretien de la maison ou qu'il plaise au Roy leur accorder quelqu'autre gratiffication annuelle pour

\footnotetext{
${ }^{43}$ Ce placet, auquel des droits de franchise sur le sel et le vin avaient été associés, fut refusé par le roi, malgré le soutien des avocats généraux.
} 
les frais de la bibliotèque comme de faire relier les livres et manuscripts et achepter ceux qui manquent

Représentent Que cette bibliothèque est d'un très grand advantage pour le public estant ouverte trois fois la sepmaine à tous ceux qui y veulent venir travailler en sorte qu'il s'y trouve toujours un nombre considérable d'estudians mais que comme par la partition des manses abbatialle et conventuelle, il ne leur a laissé que ce qui est nécessaire pour la subsistance et l'entretien de la maison, il ne leur reste plus de fonds pour fournir aux frais de l'entretenement de la bibliothèque que mesme ils ont déjà emprunté des sommes considérables pour les bastimens qu'ils ont esté obligez de faire pour agrandir le logement de cette bibliotèque en sorte qu'ils en payent actuellement plus de 1000 livres de rente.

Que cependant ils sont encore obligez a beaucoup de despense pour l'entretennement tant de la bibliotèque de leur maison que de celle qui leur a esté laissé par le feu Sieur du Bouchet conseiller lequel leur avoit aussy legué un fonds de 700 livres de rentes mais qui se trouve présentement réduites à 312 livres par les retranchements et les conversions qui ont esté faites

Raportent 31 juillet 1684 [cette date est dans la marge] un procez verbal des deux advocats généraux du Parlement lesquels comme gardes de lad. bibliotèque en ayant fait la visite confirment tout ce qui est exposé par les souscripts et estiment qu'ils doibvent se pourvoir au Roy, n'ayant pas un fonds suffisant pour fournir aux frais dont ils sont chargez.

3. REGISTRE CAPITULAIRE, 1762-1789

Arch. nat., LL 1451, fol. $13^{\mathrm{v}}$

Le 20 juillet 1764, les victorins souhaitent la construction d'une nouvelle aile de la bibliothèque afin de ne plus soustraire au public une partie importante de leurs collections.

Copie du mémoire que les chanoines de l'abbaye de Saint-Victor de Paris se proposent de présenter à Mgr l'évêque d'Orléans ${ }^{44}$ sous les auspices et la protection de Messieurs les procureurs général et avocats généraux.

De toutes les bibliothèques publiques de Paris, celle de l'abbaye de SaintVictor est la plus ancienne et une des plus célèbres, ouverte au public plus souvent et plus longtemps qu'aucune autre ; elle est aussy la plus fréquentée des gens de lettres ; elle contient une très grande quantité de livres anciens et rares, tant manuscrits qu'imprimés, dont la plus part ne se trouvent point ailleurs. Lorsque les chanoines de Saint-Victor consacrèrent leur bibliothèque à l'usage public en 1654, ils firent construire à leurs frais un bâtiment convenable. Le nombre de leurs livres s'est tellement augmenté depuis, par le bon employ qu'ils ont toujours fait des revenus affectés à leur bibliothèque et par l'attention qu'ils ont apportée à y recüeillir les livres de leurs confrères décédés, que l'ancien bâtiment autrefois trop spacieux suffit à peine aujourd'huy pour en contenir environ le tiers. Les chanoines se sont trouvés forcés de déposer tout ce surplus dans des greniers qu'on a lambrissés à une autre 
extrémité de la maison, où ils sont comme en pure perte pour le public, qui a été l'unique objet dans un établissement aussy utile. Les gens de lettres sont aussy privés par la même raison d'un recüeil considérable d'estampes et de cartes de géographie, l'une des plus précieux que l'on connaisse en Europe.

Les chanoines de Saint-Victor sentent depuis longtemps la nécessité qu'il y a d'augmenter leur bâtiment de bibliothèque et de luy donner l'étendüe suffisante pour le service du public. Toujours attentifs à saisir les occasions de se rendre utiles, ils n'eussent pas différé jusqu'à ce jour d'y pourvoir par leur propre fonds, s'il leur eût été encore possible dans cette nouvelle circonstance de prendre sur les revenus de la manse canoniale les sommes nécessaires pour une entreprise aussy dispendieuse ; mais les différentes charges et impositions ayant été considérablement augmentées depuis plusieurs années, ce n'est que par la plus exacte économie que les revenus de la maison fournissent aux dépenses et réparations annuelles. Les rentes assignées à la bibliothèque, modiques dans leur origine, ont été réduites dans ces derniers temps à moins de moitié. A peine reste-t-il aujourd'huy de quoy faire chaque année l'acquisition des livres les plus nécessaires dans une bibliothèque publique.

Les chanoines de Saint-Victor ne peuvent donc que recourir aux libéralités du Roy en faveur d'un établissement de cette nature et de cette importance pour le public. Ils se présentent pour solliciter les bienfaits de sa Majesté. Ils espèrent, Mgr, que dans un objet aussy intéressant pour les sciences, votre grandeur voudra bien les honorer de ses bontés et leur accorder sa protection pour obtenir du Roy sur l'abbaye de Saint-Victor, actuellement vacante ${ }^{45}$, ou sur telle autre qu'il luy plaira à désigner, une somme annuelle pendant certain temps, pour subvenir aux frais de l'augmentation du bâtiment de bibliothèque, selon les plans et devis que votre grandeur aura approuvés.

Ils se flattent, Mgr, que vous écouterez d'autant plus favorablement leur demande qu'ils s'y trouvent en quelque sorte autorisés par le témoignage de M. les avocats généraux Talon ${ }^{46}$ et de Lamoignon $^{47}$ dans une visite juridique que ces magistrats firent de la bibliothèque de Saint-Victor en 1684 après avoir remarqué leur satisfaction de l'attention qu'on apportoit au service du public, que vu la modicité des revenus, la maison de Saint-Victor s'adresse au Roy pour obtenir un secours qui tournera tout entier à l'avantage du public.

Les chanoines de Saint-Victor n'ajouteront rien à ces représentations. La protection, Mgr, que votre grandeur accorde à la littérature sacrée et prophane, l'attention qu'elle apporte à soutenir les études et les sciences dans l'état de splendeur où elles sont, leur sont un garant sûr que vous voudrez bien leur procurer les secours nécessaires pour faciliter le service du public et pour faire joüir les gens de lettres de la totalité d'une bibliothèque qui provient du zèle de leurs prédécesseurs, et que la libéralité de plusieurs particuliers a considérablement augmentée. Cette grace ne peut que les exciter à redoubler leurs soins pour entretenir et perpétuer un monument si précieux, et à continuer leurs vœux pour la conservation de sa Majesté et la santé et prospérité de votre grandeur.

\footnotetext{
${ }^{46}$ Je remercie Guy-Michel Leproux pour l'aide apportée ici. Denis Talon (1627-1698), devenu très jeune avocat général. Il est le fils d'Omer Talon.

${ }^{47}$ François-Chrétien de Lamoignon (1644-1709), qui fut longtemps avocat général.
} 
Luce, le prieur ${ }^{48}$

Fr. Dufresne Ducange notaire

L'affaire mise en délibération, la compagnie a agrée et statué qu'on le présenteroit à M. l'évêque d'Orléans, après en avoir prévenu M. les gens du Roy et les avoir prié d'appuyer notre demande.

Luce prieur

${ }^{48}$ Il s'agit du prieur Claude François Luce. Un victorin, qui tenait le rôle du notaire, portait sa signature à côté de celle du prieur. 